\title{
2 Die Geburt einer evolutionären Bewusstseinskultur
}

\author{
Thomas Steininger
}

Wenn wir von der Evolution des Bewusstseins sprechen, sprechen wir von uns selbst. Wir, unsere Selbst-Wahrnehmung und unsere Wahrnehmung der Welt, verändern sich. Lassen sie mich diesen Beitrag mit einer Frage beginnen - Sie ist vielleicht der direkteste Ausgangspunkt, wenn wir die Frage nach einer Bewusstseinsevolution mehr als nur theoretisch verstehen wollen. Diese Frage heißt:

„Alswer oder wasbin ich hier?“"Alswer oder waslese ich diese Zeilen?“... Nehmen Siesich einen Augenblick Zeit, Ihre eigene Antwort auf diese Frage zu finden. „Als wer oder wasbin ich hier?“

Diese Frage basiert natürlich auf einer Voraussetzung. Sie basiert auf der Annahme, dass wir, Sie und ich, die Welt immer aus einer bestimmten Perspektive sehen.

- Können wir uns unserer eigenen Perspektive, unserer eigenen Sicht auf die auf die Welt, bewusst werden?

- Können wir beginnen zu sehen, auf welche Art und Weise wir sehen?

Um das zu erreichen, hilft es, wenn wir uns selbst in einem historischen Kontext wahrnehmen.

In unserem Alltagsverständnis gehen wir oft von einer sehr vereinfachten Vorstellung aus, wenn wir darüber nachdenken, was es bedeutet, ein Mensch zu sein - als hätte das immer, zu allen Zeiten, das Gleiche bedeutet. Aber wir sind Menschen des beginnenden 21. Jahrhunderts.

- Was verbindet und was trennt uns von den Menschen des 15. Jahrhunderts?

- Was verbindet und was trennt uns von den Menschen vor 1.00o Jahren, vor 5.00o Jahren, vor 50.00o Jahren? 
Nehmen Sie sich noch einmal einen Augenblick Zeit und versuchen Sie dieser Reise in die Vergangenheit innerlich nachzugehen. Vor 50.000 Jahren existierte nicht nur eine andere Welt, wir Menschen waren damals auch ganz anders ,in der Welt“, als wir es heute sind. Genau das meinen wir, wenn wir von Bewusstseinsevolution sprechen - unser „In-der-Welt-sein“ hat sich verändert. Lassen Sie uns, soweit das in unserer Vorstellung überhaupt möglich ist, zu den Anfängen gehen, zu unseren Anfängen als Menschheit.

Vor 80o.ooo Jahren begannen wir, das Feuer zu nutzen. Die etabliertesten Schätzungen gehen davon aus, dass die ersten Feuerstellenfunde ca. 900.00o Jahren alt sind. Damals begann der Homo erectus, das Feuer zu kultivieren. Die Entdeckung des Feuers brachte uns damals neue ungeahnte Möglichkeiten. Sie revolutionierte unsere Nahrungsmittelversorgung. Von nun an waren wir in der Lage, Fleisch und Gemüse durch Kochen vorzuverdauen. Wir konnten uns viel leichter und einfacher mit Nahrung versorgen. Aber das Feuer veränderte auch die Sicht auf unsere Welt, denn es diente nicht nur dem Kochen und Garen von Lebensmitteln. Feuer gibt uns auch Licht und Wärme. In kalten Nächten konnten wir uns nun um ein Feuer scharen, um die Kälte der Nacht besser zu überstehen. Langsam begann das Feuer, unsere Vorstellung der Welt zu verändern.

Wenn wir einen Feuerplatz haben, an dem sich der Stamm oder die Horde über Jahrhunderte gewöhnt, weil wir hier die Nächte zusammen verbringen und im Laufe der Jahre das Leben miteinander teilen, vollzieht sich eine Teilung der Welt, die es vorher nicht gab. Vorher war die Welt einfach die Welt. Jetzt gab es einen geschützten Platz des Feuers. Es gab ein Hier und es gab ein Draußen. Dieses Hier hat sich erhellt, selbst in der Nacht, weil es ermöglicht, sich zu sehen. Und in der gleichen Weise, wie sich dieses Hier erhellt hat, verdunkelte sich natürlich die Nacht. Das Draußen ist dunkler geworden, während das Hier heller geworden ist.

\section{Die Geburt der Sprache}

Der Feuerplatz war auch der wichtigste Platz unserer sozialen Kontakte. Hier waren wir miteinander und tauschten uns aus - durch Laute und langsam über die Zeit auch über Worte. Wahrscheinlich fanden wir am Feuerplatz zur Sprache. Was Laute waren, wurden Worte. Ein Laut ist oft ein Signal, das in uns und in unseren Artgenossen eine bestimmte Reaktion auslöst. Ein Wort kann mehr. Wir geben den Dingen Namen wie Baum, Berg, Löwe, Gewitter oder Mammut. Aber wir können das Wort ,Löwe“ auch verstehen, wenn es keinen Löwen in unserer Umgebung gibt. Wir wissen, dass ein Löwe gemeint ist. Wir glauben aber nicht fälschlicherweise, dass dieser Löwe hier sein muss. Wir können von einem Löwen sprechen, der nur in unserer Erinnerung, nur in unserer Vorstellung existiert. Das können wir nur mit Worten, nicht mit Lauten. Welch ein Durchbruch - wir können auf einen Löwen, auf ein Mammut verweisen, das gerade in unserer Umgebung nicht existiert. Auf einmal konnten wir beginnen, uns Geschichten zu erzählen.

Es gab am Anfang keine klare Unterscheidung zwischen einem Wort und einer äußeren Realität, zwischen dem Wort ,Berg، und einem Berg. Das gab der magischen Sprache ihre Kraft. In der magischen Welt gab es keine Trennung zwischen Innen und Außen, zwischen dem, was wir mit Sprache ausdrücken, und dem, was wir heute als Außen bezeichnen, die reale Natur, der reale Berg, der reale Baum. In der entstehenden magischen Welt ist alles noch eine ungeteilte Welt, auch wenn sich diese Welt 
gerade durch die Entdeckung der Sprache zu differenzieren beginnt. Wir sind nicht mehr ungetrennt eins mit der Natur. Wir können anfangen, über sie zu sprechen.

Und unsere Sprache beginnt, ihr eigenes Leben zu entfalten. Wahrscheinlich saßen wir am Lagerfeuer und begannen, Geschichten zu erzählen - und aus Worten Sätze zu formen, und aus Sätzen Geschichten. Wir fingen an, Geschichten zu erzählen. Wir kennen noch Reste dieser ersten Geschichten. Diese alten, magischen Geschichten von der Belebtheit der Natur, von ihrer Beseeltheit, von Bachquellen, die nicht einfach Orte da draußen sind, sondern Kräfte und Wesen, die real in einer Welt existieren, in der Innen und Außen noch nicht getrennt sind.

Das waren Geschichten von den Kräften der Natur und von den Mächten, die unsere natürliche Welt durchwoben; Geschichten von unseren Ahnen und von unserem Ausgeliefertsein. So entstanden erste Formen einer sprachlichen Kultur. Heutige indigene Völker und deren Erzählungen geben uns noch einen Eindruck von dieser Welt. Auch in Resten mancher Märchen und Sagen lebt diese Welt noch fort, wie wir sie uns in unserer Frühzeit erschlossen haben.

Aber auch unsere Erzählungen entwickelten ihr eigenes Leben. Unsere Sprache formte sich. Mit neuen Worten und neuen sprachlichen Fähigkeiten dehnte sich unser Bewusstsein in neue Räume aus. Die ursprünglichen Sagen und Mythen beschäftigten sich hauptsächlich mit Kräften und den Mysterien der Natur - mit der Tierwelt. Aber auch mit unseren Vorfahren, die in dieser neuen Welt der Sprache und der Erinnerung zu einem Leben fanden, das weit über ihren physischen Tod hinausreichte. Auch heute heißt es noch in manchen afrikanischen Naturreligionen, dass jemand erst dann wirklich stirbt, wenn der Letzte gestorben ist, der sich an ihn oder sie erinnern konnte.

Erst wenn die Erinnerung an uns, die Erzählungen über uns sterben, sterben wir.

Diese Erzählungen über unsere Ahnen wurden zu einem immer größeren Teil dieses neuen Universums der Geschichten und Erzählungen. Langsam entstanden so die großen Epen der Menschheit. Unsere Sprach- und Vorstellungskraft entwickelte allumfassende Götterwelten - seien es die Götterwelten des chinesischen Kulturraums, die Götterwelten Indiens oder des Mittelmeerraums - so unterschiedlich sie sein mögen, wenn wir sie parallel lesen, ist es phänomenal zu sehen, wie sehr sich diese Epen ähneln. Kamen unsere ersten Götter und Göttinnen aus der Natur, so ähneln die Götterwelten der ersten Hochreligionen immer mehr unserer Menschenwelt, unserer Konflikte und Potenziale.

Hochreligionen, wie die griechisch-römische Religion in Europa, zeugen von diesem neuen In-der-Welt-sein - das mehr und mehr auch eine Welt der Menschen war. Aber genauso wie die schamanischen Religionen waren auch diese Hochreligionen teil unseres Ausdrucks einer durch und durch belebten Welt. Alles war belebt. Jede Quelle hatte ihre Göttin, ihren Gott, ihren Geist, ihren Spirit. Jede menschliche Qualität fand ihren Vertreter im Olymp. Unser Universum, unser Kosmos war eine durch und durch belebte und beseelte Welt.

\section{Die Entdeckung des Absoluten}

Es gab einen Zeitpunkt in unserer Geschichte, da haben sich unsere Mythen radikal verändert. Erstaunlicherweise geschah das rund um den Erdball ziemlich zur gleichen Zeit, in einem Zeitraum von knapp 50o-70o Jahren. Auf einmal kam sowohl im chinesischen Kulturkreis als auch in Indien und im Kulturkreis des Mittelmeerraums 
etwas zur Sprache, das es vorher so nicht gab - etwas Absolutes, eine Dimension, die die Welt übersteigt oder ihr zugrunde liegt. Diese Dimension wurde in den verschiedenen kulturellen Kontexten Chinas, Indiens oder des Mittelmeerraumes in den jeweiligen kulturellen Kontexten anders erschlossen. Sie hatte andere Namen, aber diese neuen Erzählungen hatten eine grundlegende Cemeinsamkeit: Sie sprachen von etwas Absolutem.

In diesem historischen Zeitraum - der deutsche Philosoph Karl Jaspers hat dieser Zeit einen Namen gegeben, er sprach von der Achsenzeit - gab es herausragende Persönlichkeiten. Man könnte sagen, es waren Vorreiter eines neuen Bewusstseins, die begannen, von dieser absoluten Dimension zu sprechen. In China waren es Menschen wie Laotse, in Indien Shankara oder Buddha Gautama, im Mittelmeerraum waren es jüdische Propheten wie Elias, aber auch griechische Philosophen wie Pythagoras oder Plato gehören zu diesen herausragenden Menschen. Die einen sprachen vom Tao, die anderen von Brahman, die jüdischen Propheten nannten es den nicht nennbaren Gott. Plato sprach vom absoluten Guten. Sie alle sprachen von etwas, das jenseits der relativen Welt war. Die Interpretationen dieses Absoluten war in den Kulturkreisen und ihren Mythenwelten unterschiedlich. Aber überall kam es in dieser Achsenzeit zu einem radikalen Umbruch unserer Bewusstseinswelt. Neben dem allumfassenden, belebten Universum, wie wir es in unserer Sagen- und Mythenwelt beschrieben, gab es auf einmal etwas, das alles überstieg, das man nicht einmal wirklich benennen konnte, weil dessen Grundqualität etwas Unaussprechbares war.

Aus heutiger Sicht könnte man vielleicht sagen, wir wurden uns damals des Bewusstseins selbst bewusst, jenseits seiner relativen Inhalte. Hegel nannte diese Dimension einmal das absolute Bewusstsein, aber auch das ist nur eine Interpretation von etwas, das unaussprechlich bleibt. All die Götterwelten, all die Sagenwelten, all die Welten, die wir beschreiben, sind Inhalte unseres Bewusstseins. Jenes absolute Bewusstsein ist vielleicht der Raum, in dem sich alle Inhalte überhaupt erst zeigen können. Buddha Gautama nannte es die Leere, das Nichts. Die großen jüdischen Propheten sprachen vom Gott, dem man keinen Namen geben darf.

Diese großen Männer und Frauen haben etwas in die Sichtbarkeit gebracht, das die Kulturgeschichte der Welt radikal verändern sollte. Ihre Einsichten lösten kulturelle Revolutionen aus. Sowohl im klassischen Indien wie auch im christlichen Mittelalter gingen ganze Generationen junger, gebildeter Menschen in die Berge oder ins Kloster, um der relativen Welt zu entsagen und um dem Absoluten - sei es der jüdisch/ christliche Gott oder die indische Erleuchtung - Raum und Bedeutung zu geben. Es gibt Berichte aus dem frühen Hochmittelalter, die beklagten, dass die Besten der Generation nicht zur Verfügung standen, um die Gesellschaft oder die Wirtschaft zu entwickeln - sie gingen ins Kloster.

Aber nicht nur unsere Mythen haben sich in der Achsenzeit grundlegend verändert. Auch unser Verhältnis zur Sprache selbst veränderte sich damals grundlegend. Mit Sprache kann man Geschichten erzählen und das taten wir von Anfang an. Sprache hat aber auch ihre eigene innere Struktur, eine eigene, innere Logik. Und es gab einen bestimmten Zeitpunkt, an dem wir begannen, diese innere Struktur, die Logik von Sprache, mehr und mehr zu sehen und zu verstehen. Mit diesem neuen Verständnis entdeckten wir etwas Neues - den bewussten Umgang mit Logik und Rationalität. Das herausragendste Beispiel für diese Entdeckung der Rationalität war das Griechenland des 6. und 5. vorchristlichen Jahrhunderts. Was damals im antiken Griechenland 
neu entstand, nennen wir heute die Geburt der Philosophie. Auf einmal standen nicht mehr Mythen und Epen im Mittelpunkt unserer Aufmerksamkeit. Philosophen wie Thales und Diogenes ging es um eine rationale Form des Nachfragens: Was ist wahr, was ist falsch?

Die Geburt der griechischen Philosophie war die Geburt einer bewussten Rationalität. Das war eine andere Art zu sprechen, eine andere Art zu denken. Diese Geburt der Rationalität gab es auch auf dem indischen Kontinent und auch in der chinesischen Philosophie, aber in Griechenland entwickelte sie eine eigene Dynamik, die Europa über die Jahrhunderte tief prägen sollte. Sie hat die Art und Weise, wie wir in der Welt sind, radikal verändert.

Jetzt war nicht mehr die Bildkraft der Sprache allein das Ausschlaggebende. Zu ihr gesellte sich die Überzeugungskraft des Arguments. Zur Bildkraft des Mythos gesellte sich die Kraft des Arguments, die Überzeugungskraft des Logos.

\section{Das Ich}

Es gibt noch einen Entwicklungsstrang unserer Bewusstseinsgeschichte als Menschheit, der im klassischen Griechenland seinen Ausgangspunkt nahm. Die Sprache, der sprachliche Austausch, entstand in Situationen wie dem Lagerfeuer der Sippe, im Kollektiv eines Stammes oder eines Volkes. Um zu sprechen, braucht es aber Sprecher, einzelne Sprecher. Im griechischen Theater konnte man schon den Übergang zwischen der Stimme des Chors und der einzelnen Charaktere sehen - wir wurden uns langsam bewusst, dass wir Individuen sind. Diese Bewusstwerdung, in der wir erkannten, dass wir nicht nur einer Cruppe angehören, sondern auch Individuen sind, hat vielleicht niemand so eindringlich beschrieben, wie der Schweizer Psychologe und Mystiker C.G. Jung. Er stellte unsere Bewusstseinsgeschichte als Individuationsprozess dar, durch den wir uns als Gattung - aber auch als Individuen - weiterentwickeln.

Es gab in diesem Individuationsprozess verschiedene wichtige Stationen. In unserer abendländischen Geschichte mit ihren jüdisch-griechischen Wurzeln spielt der Kirchenlehrer Augustinus eine entscheidende Rolle. Wer je Augustinus' Bekenntnisse gelesen hat, sieht in diesem Buch, das in manchen Teilen schon Elemente der Tiefenpsychologie vorwegnimmt, die Auseinandersetzung eines einzelnen Suchenden, dessen Beziehung zum Göttlichen aus einer fast modernen Ich-Du-Beziehung lebt. Es ist diese radikale Ich-Du-Beziehung, die gerade auch mit Augustinus zu einer speziellen Charakteristik der abendländischen Spiritualität wurde. Dem absoluten Du steht ein suchendes Ich gegenüber. Und dieses Ich wird zu einem treibenden Motor der europäischen Kulturgeschichte.

Nach Augustinus' Bekenntnissen ist einer der nächsten Meilensteine dieser Individuationsgeschichte wohl Martin Luther. Für Martin Luther gibt es keine vermittelnde Rolle der Kirche und der Priester mehr. Der „freie Christenmensch“, der jetzt Gottes Wort in seiner eigenen Sprache lesen kann, braucht nichts als diese Bibel und seine ganz persönliche Beziehung zum absoluten $\mathrm{Du}$.

Bald sollte aber dieses Du verloren gehen. Denn im Übergang von der Reformation zur Aufklärung rückt Gott in weite Ferne. Was bleibt, ist ein neuer absoluter Grund, ein neuer Ausgangspunkt, von dem aus wir in der Welt sein können: wir selbst. René Descartes formulierte als Erster diese moderne Bewusstseinsrevolution mit seinem berühmten Satz: „Cogito ergo sum. Ich denke, also bin ich.“ Doch dieser Satz wird 
oft missverstanden. „Cogito“ meint mehr als nur Denken. Man könnte ihn auch übersetzen mit:

„Ich bin bewusst, ich bin denkend bewusst, also bin ich.“

Mit Descartes verschiebt sich unsere Wahrnehmung der Welt noch einmal. Man könnte auch von einer radikalen Umkehrung sprechen. Waren wir vorher auf eine gewisse Art und Weise naiv einfach ,in der Welt“ und war dieses In-der-Welt-sein etwas Unangezweifeltes, so begann Descartes und mit ihm die moderne Philosophie, an dieser naiven Annahme zu zweifeln.

Gibt es die Welt überhaupt, und wenn ja, kann ich sie erkennen?

Descartes meinte, das Einzige, an dem ich nicht zweifeln kann, ist dieses „cogito“, dieses „Ich denke, ich bin denkend bewusst“, denn selbst wenn ich zweifle, denke ich. Mit dieser Kehrtwende ist die Basis unseres In-der-Welt-seins keine lebendige Welt mehr, auch keine absolute Dimension, sondern unser Ich. Und die Welt ist da draußen - ich kann daran zweifeln, ob sie überhaupt existiert.

Viele Denker, die die menschliche Kultur- und Bewusstseinsgeschichte beschrieben haben, sehen diese Wende als eine Entfremdungsgeschichte, eine Trennungsgeschichte. Aber sie ist andererseits auch eine Freiheitsgeschichte. Dieses Ich, das wir in Europa zum Ausgangspunkt für unsere Welt gemacht haben, ist der einzige Ausgangspunkt, von dem aus es so etwas wie die Freiheit des Willens und damit echte Verantwortung geben kann.

Diese Dimension von Freiheit, die Freiheit des Willens, wurde nirgendwo stärker kultiviert als in Europa. Sie macht meines Erachtens auch die Würde der europäischen Moderne aus: Wir haben die Freiheit, uns entscheiden zu können. Die Freiheit, als mündige Menschen - als Mündige „befreit aus selbst verschuldeter Unmündigkeit“, wie es Kant formuliert - entscheiden zu können und damit auch entscheiden zu müssen. Diese Form von Entscheidungsfreiheit ist die Grundlage unserer Menschenrechte, unserer Menschenwürde und auch die Grundlage unseres Verständnisses von Demokratie. Beides - sowohl die Entfremdungsgeschichte als auch die Entwicklung unseres freien Willens, das Erkennen unseres freien Willens als Entscheidungsmöglichkeit - ist Teil dieser Bewusstseinsgeschichte der europäischen Moderne.

Ich möchte noch einen Punkt zu diesem kurzen Abriss der Bewusstseinsgeschichte hinzufügen, der noch sehr jung ist. Dieser Durchbruch ist eigentlich erst in den GoerJahren der 20. Jahrhunderts zu seiner Blüte und zu seinem vollen Durchbruch gekommen. Ich spreche von unserer postmodernen Kultur. In der Entwicklung der Moderne haben wir gelernt, die Welt aus einer Ich-Perspektive wahrzunehmen, die rational fundiert ist. Dies zeigte sich in der Malerei der Renaissance durch die Entdeckung der Perspektive, die nichts anderes ist, als die Entdeckung der Ich-Perspektive. Die Perspektive des Malers und des Betrachters wurde im Gemälde sichtbar; sie wurde sozusagen mitgemalt. In der Postmoderne wurde diese radikale Ich-Perspektive der Moderne aufgebrochen und wir waren auf einmal in der Lage oder haben gelernt, mehrere Perspektiven gleichzeitig zu sehen. In der Kunst ist dieser Durchbruch durch Künstler wie Picasso sichtbar geworden, aber es geht hier nicht allein um Kunst. Wenn man den Aufbruch der zweiten Hälfte des 20. Jahrhunderts in einem Satz zusammenfassen will, dann ist das vielleicht die Entdeckung der Multiperspektivität. Alles kann aus verschiedenen Gesichtspunkten gesehen werden, nicht nur der des weißen, europäischen Mannes. 


\section{Und nun?}

Wir sind jetzt vielleicht an einem Punkt, wo die Postmoderne ihren Höhepunkt schon überschritten hat; es ist eine Postmoderne, die auch ihre Schattenseiten, wie Relativismus und Narzissmus, sieht. An diesem Punkt stellen sich folgende Fragen:

- Was ist der nächste Schritt unserer Bewusstseinsevolution?

- Welches neue In-der-Welt-sein bietet echte Antworten auf die Fragen unserer Zeit?

Ich glaube, was wir heute neu wahrnehmen können, ist genau das, was ich versucht habe, hier zu beschreiben: Wir erkennen in der Menschheitsgeschichte einen großen Entwicklungsstrang, in dem sich unser Bewusstsein entwickelt. Wir sind Teil und Ausdruck dieses sich entwickelnden Bewusstseins. Lassen Sie mich ich die Frage, die ich Ihnen am Anfang dieses gestellt habe, noch einmal in diesem Kontext stellen:

\section{„Als was oder wer bin ich hier?“}

Und nehmen Sie sich einfach wieder einen Augenblick Zeit dafür - unter dem Eindruck dessen was sie gerade gelesen haben dann: Als was oder wer sind Sie hier? Wahrscheinlich zeigen sich verschiedene Dimensionen: Es gibt die Dimension Ihrer Individualität. Es gibt einen kulturellen Kontext und einen Zeitkontext, es gibt aber auch die Dimension eines Bewusstseins, unseres menschlichen Bewusstseins, das beginnt, sich dieser Entwicklungsdynamik des Bewusstseins und der Kultur in uns selbst bewusst zu werden. Denn das, was unser Menschsein ausmacht, ist auch dieser große kreative evolutionäre Impuls, der uns bis zu diesem Punkt in unserer Bewusstseinsgeschichte gebracht hat.

Auf einmal kommt eine Dimension in die Sichtbarkeit, die nicht nur meine Individualität betrifft, obwohl diese davon stark betroffen ist: unsere menschliche Erfahrung als Ganzes. Wir sind ein Prozess, der sich über Hunderttausende von Jahren entfaltet hat und wir sind - und das ist vielleicht der außergewöhnliche Augenblick unserer Zeit - an dem Punkt, wo sich dieser Prozess seiner selbst bewusst wird. Noch nie zuvor haben wir diesen Bewusstwerdungsprozess, den ich vorhin zu schildern versuchte, so bewusst wahrnehmen können, wie ihn Menschen wie Jean Gebser, Sri Aurobindo, Andrew Cohen oder Ken Wilber beschreiben .

Was ändert sich, wenn unsere Bewusstseinskultur auf einmal zu dieser Dimension ihrer eigenen Entwicklung, ihrer eigenen Entfaltung aufwacht?

Auf einmal wird sichtbar, dass diese Dimension etwas ist, für das wir eine gemeinsame Verantwortung übernehmen können. Wir können nur Verantwortung übernehmen für etwas, dessen wir uns bewusst sind. Aber in dem Augenblick, in dem wir sehen, dass hier ein riesiger Prozess eines sich entfaltenden Bewusstseins wirkt, müssen wir auch erkennen, dass wir gefragt sind.

Wie verhalten wir uns zu dieser Erkenntnis, die sich uns gerade heute stellt?

Wenn Menschsein auch bedeutet, dass wir uns dieses ganzen Prozesses bewusst werden und Verantwortung dafür übernehmen, ihn bewusst weiterzugestalten.

Ändert sich dann die Art und Weise, wie wir In-der-Welt-sind?

Wenn das gelingt, dann stehen wir am Anfang einer neuen, evolutionären Bewusstseinskultur. 


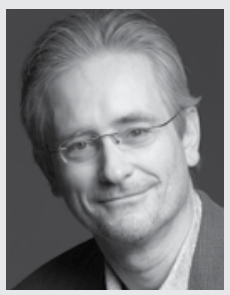

Dr. Thomas Steininger

studierte Philosophie an der Universität Wien und schrieb Ende der 80erJahre als einer der Ersten im deutschsprachigen Raum eine Dissertation unter Verwendung der integralen Theorie Ken Wilbers. Er arbeitete für das österreichische Radio (ORF-Ö1) und als freier Journalist mit dem Schwerpunkt auf spirituellen Themen. Herr Steininger lehrte beim Masters-Programm für "Conscious Evolution" am Graduate Institute in Connecticut/USA in Zusammenarbeit mit Don Beck, Susanne Cook-Greuter, Allan Combs u.a. Seit fast zwanzig Jahren wirkt er an der Entwicklung der Evolutionären Dialoge mit. Dr. Steininger ist Leiter von EnlightenNext Deutschland/Schweiz und Herausgeber der Zeitschrift EnlightenNext Impulse und Moderator von Radio EnlightenNext. 